\title{
Escape from attracting sets in randomly perturbed systems
}

\author{
Christian S. Rodrigues, ${ }^{1,2, *}$ Celso Grebogi, ${ }^{2}$ and Alessandro P. S. de Moura ${ }^{2}$ \\ ${ }^{1}$ Max Planck Institute for Mathematics in the Sciences, Inselstr. 22, 04103 Leipzig, Germany \\ ${ }^{2}$ Department of Physics and Institute for Complex Systems and Mathematical Biology, King's College, University of Aberdeen, \\ Aberdeen AB24 3UE, United Kingdom \\ (Received 15 April 2010; revised manuscript received 20 August 2010; published 20 October 2010)
}

\begin{abstract}
The dynamics of escape from an attractive state due to random perturbations is of central interest to many areas in science. Previous studies of escape in chaotic systems have rather focused on the case of unbounded noise, usually assumed to have Gaussian distribution. In this paper, we address the problem of escape induced by bounded noise. We show that the dynamics of escape from an attractor's basin is equivalent to that of a closed system with an appropriately chosen "hole." Using this equivalence, we show that there is a minimum noise amplitude above which escape takes place, and we derive analytical expressions for the scaling of the escape rate with noise amplitude near the escape transition. We verify our analytical predictions through numerical simulations of two well-known two-dimensional maps with noise.
\end{abstract}

DOI: 10.1103/PhysRevE.82.046217

PACS number(s): 05.45.Ac, 61.43.Hv, 02.50.Ey

The escape of trajectories from attracting sets due to the effect of noise has been a central issue in various branches of science for a long time. From a fundamental perspective, the study of the dynamics of escape includes the fundamental work by Arrhenius on chemical reactions [1], passing through ideas of Kramers from the 1940s [2], the escaping on chaotic dynamics [3-6] to very recent work on statistical mechanics [7]. Notwithstanding this long history, an important case has mostly been neglected, namely, the dynamics under bounded noise. In fact, the vast majority of theoretical works in this area is heavily dependent on the assumptions of unbounded noise, almost always assumed to have a Gaussian distribution $[3,7,8]$. Thus, they are not applicable to other cases. In particular, very little is known about the dynamics of systems with escape in the presence of bounded noise. As for many applications a bounded perturbation is arguably more realistic than unbounded ones, this is an important gap in our understanding. For example, in neuroscience one may be interested in the minimum energy for bursting to take place [9], in geophysics one may consider the overcoming of some potential barrier just before an earthquake [10], or the critical outbreak magnitude for the spread of epidemics [11], among others.

In this paper, we investigate escape in dynamics perturbed by bounded noise using a different approach. We describe the noisy dynamics in terms of a family of random maps, whose iteration gives rise to a discrete Markovian process with transition probability supported in the neighborhood of the points generated by the iteration of the deterministic dynamics. The dynamics near an attractor is then described using the formalism of conditionally invariant measures. We show that the dynamics of the escape is determined by the measure of a subset $I_{\partial}$ of the phase space, which we call conditional boundary. It consists of those points lying close enough to the basin boundary such that they are subject to escape under the effect of random perturbation in one iteration of the map, and intersecting the support of the conditionally invariant

\footnotetext{
*christian.rodrigues@mis.mpg.de
}

measure of the system. Therefore, the dynamics is mapped onto the dynamics of a closed system with a hole $-I_{\partial}$ being the hole. We show that there is a minimum noise amplitude for the escape to take place, which is the critical noise amplitude $\xi_{c}$ that makes $I_{\partial}$ nonempty. We show that the mean escape time is determined by the measure of the conditional boundary and use this to derive a power-law relation between the average escape time $\langle T\rangle$ and the noise amplitude $\xi$, for $\xi$ close to (and higher than) $\xi_{c}$ :

$$
\langle T\rangle \approx\left(\xi-\xi_{c}\right)^{-\alpha}
$$

where $\alpha$ depends on the dimension of the system. We show that for dimension $2, \alpha=3 / 2$, and we verify that this prediction is correct by comparing with the results of numerical simulations for two maps from different families. This result is independent of any particular system and holds universally for bounded noise. Equation (1) is in contrast with the case of Gaussian noise, where an exponential scaling is observed [1-8]. Furthermore, although the noise-induced escape from attractors may seem to be very different from that of systems undergoing a boundary bifurcation, we show that our approach allows us to establish a connection between these two processes and to explain why we find the same time scaling in both cases.

To get started, first consider a deterministic dynamics $x_{n+1}=f\left(x_{n}\right)$ given by the iteration of the map $f$, a smooth function whose inverse is differentiable, in the phase space of the system $M$ (i.e., the iteration of a diffeomorphism $f: M \rightarrow M)$. Our main focus will be on invariant subsets $\Lambda$ of $M$ which attract their neighboring points, that is, $f^{n}(x)$ tends to $\Lambda$ as $n \rightarrow \infty$; these are the attractors of the system. The basin of attraction of $\Lambda$ is the open set $W^{s}(\Lambda)$, the set of points eventually coming close and converging to $\Lambda$. The next ingredient is the boundary of the basin of attraction, a zero Lebesgue measure ergodic component of the phase space which we denote by $\partial$.

We shall consider a random perturbation of the deterministic system introduced above $[12,13]$, so that our perturbed system is described by a family of random maps, which in our context can be written as 
(a)

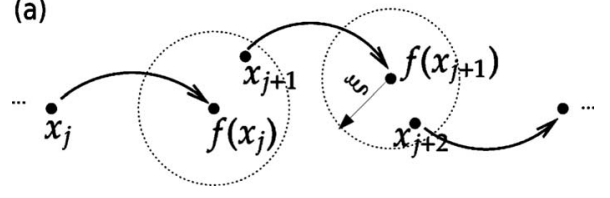

(b)

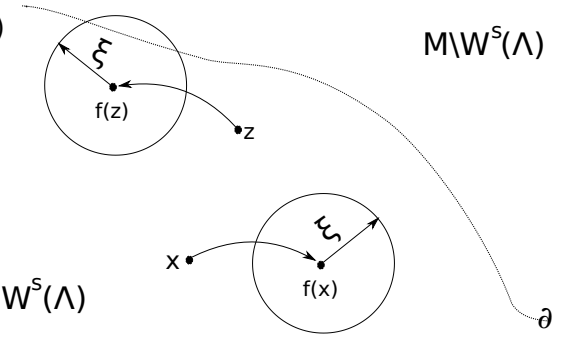

FIG. 1. (a) Illustrative picture of the perturbed dynamics with amplitude of noise $\left\|\varepsilon_{j}\right\|<\xi$. (b) A basin of attraction $W^{s}(\Lambda)$ and its basin boundary $\partial$ (dashed line). We illustrate that the iteration $z \mapsto f(z)$ from the point $z$ initially in $W^{s}(\Lambda)$ brings the orbit within a distance $\xi$ from the boundary. Therefore, the random perturbation applied to $f(z)$ with some $\|\varepsilon\|<\xi$ could push the random orbit outside the basin. On the other hand, for the point $x \in W^{s}(\Lambda)$ the iteration $x \mapsto f(x)$ brings it farther than the maximum perturbation $\xi$ away from the boundary $\partial$. Therefore, in our illustration $z \in \widetilde{I}_{\partial}$, but $x \notin \widetilde{I}_{\partial}$.

$$
F\left(x_{j}\right)=f\left(x_{j}\right)+\varepsilon_{j},
$$

with $\left\|\varepsilon_{j}\right\|<\xi$, where $\varepsilon_{j}$ is the vector of random noise added to the deterministic dynamics at the iteration $j$, and $\xi$ is its maximum amplitude. In this way, $F$ is a continuous application [14]. We illustrate this in Fig. 1(a). As it is shown in the picture, the perturbed dynamics can be thought as follows. Imagine that we iterate the point $x_{j}$ by the deterministic system $f$. Then, let say that at the moment that we take $f\left(x_{j}\right)$ to evolve our dynamics again, we make a small error given to our limited precision. Nevertheless, we can assure that the error is always less than $\xi$. Then we ask whether the attractors for the system with no error are still attractors when a small error is considered. If so, how large can our error be such that the attractors will still be preserved? Above this threshold, how does the escape of orbits scale? These natural extensions of deterministic processes are exactly the sort of problems we shall be interested in.

From the probabilistic perspective, the idea of orbits converging to some attractor is represented by the concept of physical [or Sinai-Ruelle-Bowen (SRB)] measures $[13,15]$. Suppose initially that we compute the time average, $\frac{1}{n} \sum_{j=0}^{n-1} f^{j}(x)$, along the orbit for a given initial condition as $n$ evolves. This quantity, the time average, is expected to converge to some invariant value as the orbit approaches the attractor. If we extend it for a large number of initial conditions, then the time averages that we compute along different orbits are also expected to converge to an invariant value as these orbits approach the attractor. It turns out that such value defines the so-called SRB measure, which characterizes the attractor. The set of initial conditions that we chose for computing such time averages along the orbits is exactly what we call the basin of the measure, which we represent by
$\mathcal{B}(\mu)$. Since we want to statistically characterize the behavior of the attractor, it is desirable for the set of initial conditions for which we compute such time averages to have positive Lebesgue measure. That is, there is a non-negligible number of initial conditions whose time averages along the orbits converge to such invariant quantity and thus characterize the attractor. Therefore, we say that a measure is physical if its basin has positive Lebesgue measure. More precisely, the basin $\mathcal{B}(\mu)$ of such measure is the set of points whose time averages along the orbits weakly converge to the space average, $\lim _{n \rightarrow \infty} \frac{1}{n} \sum_{j=0}^{n-1} \varphi\left(f^{j}(x)\right)=\int \varphi d \mu$, where $\varphi$ represents our measurements of an observable [16]. Here, a physical measure of a set $A \subset M$ is an invariant ergodic probability measure $\mu$ supported on $A$. Therefore, such measures are closely related to the so-called natural measures. Note that as a consequence of the Birkhoff's ergodic theorem,

$$
\mu(\mathcal{B}(\mu))=1 \text {. }
$$

In other words, Eq. (3) just says that we are normalizing such measure on the total set of initial conditions whose time averages converge to the invariant one. For most known cases the basin of the physical measures supported on the attractors coincides with the basins of the attractors [13], so we assume here that $W^{s}(\Lambda)=\mathcal{B}(\mu)$. This is known as the basin property.

Despite the complications introduced by the presence of noise in the dynamics, for continuous random maps with small amplitude of perturbation an invariant probability measure is guaranteed to exist. This is due to the KrylovBogolubov theorem [17], which ensures that every continuous application in a compact measurable space has an invariant probability measure. For randomly perturbed systems, we expect that the perturbed orbit will densely fill up the neighborhood of the attractor. This property is called random transitivity [12], and under general conditions it can be shown that these measures are ergodic [12]. As an example, we can think of an attractive fixed point for a deterministic system and the perturbed version of that system. In the perturbed dynamics, there is a density of probability around the fixed point. When we increase the amplitude of the noise, the density of probability becomes more spread around the original fixed point. Conversely, decreasing the amplitude of noise, the support of the invariant measure tends to the point attractor.

Now consider a subset $\widetilde{I}_{\partial}$ of the phase space neighboring the basin boundary, defined by the set of points $x$ whose $f(x)$ is within a distance $\xi$ from some point in the boundary $\partial$,

$$
\widetilde{I}_{\partial}=\{x \in M ; B(f(x), \xi) \cap \partial \neq \emptyset\},
$$

where $B(f(x), \xi)$ is the ball of radio $\xi$ around $f(x)$. This is illustrated in Fig. 1(b). In addition to being close enough to the boundary, another important condition for the escaping process to take place is that the intersection of the support of the invariant physical measure of the system under stochastic perturbation, supp $\mu_{\xi}$, overlaps the set $\widetilde{I}_{\partial}$. That is, the density of probability around the attractor needs to be spread over a region close enough to the boundary. We thus define the set $I_{\partial}$ by 


$$
I_{\partial}=\widetilde{I}_{\partial} \cap \operatorname{supp} \mu_{\xi}
$$

Because the noise is bounded, for very small noise amplitudes $I_{\partial}=\emptyset$. But as the amplitude of the noise is increased, we expect that for a certain critical amplitude $\xi=\xi_{c}$, we have $I_{\partial} \neq \emptyset$, and escape takes place for any $\xi>\xi_{c}$. We call $I_{\partial}$ the conditional boundary. The importance of $I_{\partial}(\xi)$ stems from the fact that it represents the set of points in which one iteration of the map $f$ can potentially send close enough to the boundary $\partial$, such that a random perturbation with amplitude $\xi$ may send them out of the basin of attraction. The dynamics of escape is thus governed by this set, and it can be understood as a hole which sucks orbits from the basin if they land on it.

Due to property (3), we have that $I_{\partial} \neq \emptyset$ for $\xi>\xi_{c}$, and thus $\mu\left(I_{\partial}\right)>0$. We can therefore think of the system with $\xi>\xi_{c}$ as a closed system with a hole (or leak) [18], where $I_{\partial}$ plays the role of the hole. The idea of a system with a hole have been used in different context before, for example [18]. Furthermore, note that the measure $\mu\left(I_{\partial}\right)$ is not in fact invariant because of the loss caused by escape. However, it is possible to describe such escape problem in terms of conditionally invariant measure $[18,19]$. We say that a probability measure $\mu_{c}$ is conditionally invariant with respect to $F$ if

$$
\frac{\mu_{c}\left(F^{-1}(X)\right)}{\mu_{c}\left(F^{-1}(A)\right)}=\mu_{c}(X),
$$

for every measurable subset $X \subset A$, where $A \subset M$ is a noninvariant region of the phase space $M$. The conditional measure is defined in a way that, for each iteration, when the set $A$ loses a fraction of its orbits to the hole, we renormalize its measure by what remains in $A$. It is defined in terms of preimages (or more generally using push forward measures [18]). In our context, the hole is the set $I_{\partial}$, and therefore $\mu_{c}\left(I_{\partial}\right)>0$ is preserved by the dynamics because of the compensation factor $\mu_{c}\left(F^{-1}(A)\right)[18,20]$. Due to the random transitivity of the conditionally invariant domain, we expect no particular dependence on the density of points in $A$. In this case, a random trajectory diffuses through the support of $\mu_{c}$, until it eventually comes inside $I_{\partial}$. Once in this set, there is a probability that it will permanently escape. Indeed it is the measure of $I_{\partial}$ which controls the escape rate. Because we are interested in the regime of $\xi \gtrsim \xi_{c}$, hence small leaks, we can assume [20]

$$
\mu_{c}\left(I_{\partial}\right)=\mu\left(I_{\partial}\right)>0 .
$$

Since there is a certain probability that a particle escapes if it falls into $I_{\partial}$, we expect from Kac's lemma [21] that the average escape time satisfies

$$
\langle T\rangle \propto \frac{1}{\mu\left(I_{\partial}\right)} .
$$

Rigorously proving the existence of and calculating $\mu\left(I_{\partial}\right)$ is not an easy task. We use here a heuristic approach to obtain the scaling of $\mu\left(I_{\partial}\right)$ for $\xi$ close to $\xi_{c}$ [22]. For simplicity we focus on the two-dimensional case. For $\xi<\xi_{c}$, we expect the probability distribution to be relatively concentrated on the attractor, thus having support with Lebesgue (a)

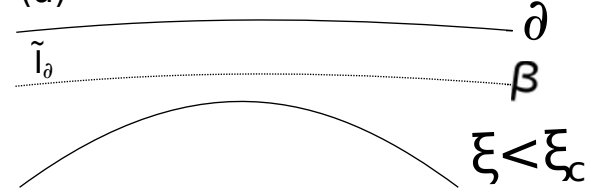

(b)

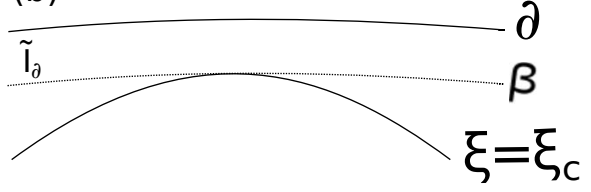

(c)

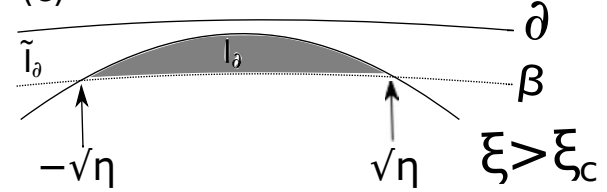

FIG. 2 . We illustrate the basin boundary $\partial$, the region $\tilde{I}_{\partial}$ whose edge is the dashed line $\beta$, and the boundary of the support of the invariant measure for the perturbed system (the parabolic line). In (a), $\xi<\xi_{c}$; thus, $I_{\partial} \cap \operatorname{supp} \mu_{\xi}=\emptyset$. In (b), $\xi=\xi_{c}$, the supp $\mu_{\xi}$ is tangent to the limit of $\tilde{I}_{\partial}$. In (c), $\xi>\xi_{c}$. As a consequence, $I_{\partial} \cap \operatorname{supp} \mu_{\xi}$ $\neq \emptyset$; therefore, $I_{\partial} \neq \emptyset$ (shadowed area), which implies $\mu\left(I_{\partial}\right)>0$.

measure (area) smaller than that of the basin of attraction. As a first approximation, we image the edge of the support as being a smooth closed curve. As $\xi$ is increased, the mean radio of the distribution grows, but it needs to be less than that of the basin of attraction; otherwise, we would have the invariant measure supported outside the basin of attraction. When $\xi \gtrsim \xi_{c}$, we picture it as a generic intersection of two curves of different radios. Therefore, the portion of the edge of the support of the invariant measure lying within $\widetilde{I}_{\partial}$ is locally well approximated by a parabola (curved line in Fig. 2 ). For $\xi \gtrsim \xi_{c}$, we have $I_{\partial} \neq \emptyset$; thus, $\mu_{c}\left(I_{\partial}\right)>0$. Recalling Eq. (7), it gives us that $\mu\left(I_{\partial}\right)>0$.

To estimate the measure of the hole, define

$$
\eta=\xi-\xi_{c}
$$

The top curve encompassing the shadowed area in Fig. 2(c) is then well approximated by

$$
S=\eta-\beta^{2}
$$

in the appropriate units. $S$ intersects $\beta$ in two points, namely, $-\sqrt{\eta}$ and $\sqrt{\eta}$. Because we assumed the basin property to hold, in other words, we normalized the measure and assumed the basin of the measure to be equal to the basin of attraction of the deterministic system, Eq. (3) also tells us that the probability measure of the hole is proportional to its Lebesgue measure, the area encompassed by $\beta$ and $S$ within the interval $[-\sqrt{\eta}, \sqrt{\eta}]$ - the shadowed area in Fig. 2(c). We can calculate it as 


$$
\begin{aligned}
& \mu\left(I_{\partial}\right) \approx \int_{-\sqrt{\eta}}^{\sqrt{\eta}} S d \beta=\frac{4}{3} \eta^{3 / 2}, \text { and thus } \\
& \mu\left(I_{\partial}\right) \approx\left(\xi-\xi_{c}\right)^{3 / 2} .
\end{aligned}
$$

Applying Kac's lemma, we obtain Eq. (1) with $\alpha=3 / 2$. Note that although we use the approximation to describe the boundary $\partial$ locally as a smooth curve, it might actually be fractal. Therefore, if the random orbit falls into $I_{\partial}$, there is only a probability that it will escape due to the fractal property of $\partial$. This fact is subtly incorporated by Eq. (8) in the proportionality rather than the equality to the inverse of the mean escape time.

In order to check this prediction we numerically obtained the scaling of the distribution of escaping times with amplitude of noise for two distinct two-dimensional systems. The first perturbed systems we have chosen was the randomly perturbed single rotor map [23], defined by

$$
F\left(\begin{array}{c}
x_{j} \\
y_{j}
\end{array}\right)=\left(\begin{array}{c}
x_{j}+y_{j}(\bmod 2 \pi) \\
(1-\nu) y_{j}+4 \sin \left(x_{j}+y_{j}\right)
\end{array}\right)+\left(\begin{array}{c}
\varepsilon_{x_{j}} \\
\varepsilon_{y_{j}}
\end{array}\right),
$$

where $x \in[0,2 \pi]$ and $y \in \mathbb{R}$, and $\nu$ represents the dissipation parameter. As a second testing system, we have chosen the perturbed dissipative Hénon map, in the form

$$
G\left(\begin{array}{c}
x_{j} \\
y_{j}
\end{array}\right)=\left(\begin{array}{c}
1.06 x_{j}^{2}-(1-\nu) y_{j} \\
x_{j}
\end{array}\right)+\left(\begin{array}{c}
\varepsilon_{x_{j}} \\
\varepsilon_{y_{j}}
\end{array}\right),
$$

where $x$ and $y$ are real numbers and, again, $\nu$ represents the dissipation parameter. We used $\nu=0.02$ for both maps, as for such value they present very rich dynamics [24]. We also assumed, for the purposes of this numerical experiment, the noise to be uniformly distributed in each variable; but we stress that this is just a numerical convenience. For each map, we computed the time that random orbits took to escape from their respective main attractors for a range of noise amplitudes. In each case, the mean escape time was obtained for $10^{3}$ random orbits for each value of $\xi$. The results are shown in Fig. 3. For the parameter used here, we obtained $\xi_{c}=0.086 \pm 0.006$ for the perturbed rotor map and $\xi_{c}=0.021 \pm 0.002$ for the perturbed Hénon map, which is shown in the insets. In both cases, we obtained a good agreement between our simulations and the predictions of our theory for a range of decades. An important remark is regarding the precision of $\xi_{c}$. For the one-dimensional case, for example, it has been proved that similar power laws in the unfolding parameters are in fact lower bounds for the average escape time scale [19]. Therefore, even from the numerical perspective, it is difficult to accurately estimate the value of $\xi_{c}$. Indeed, in our case we observe when increasing $\xi$ near $\xi_{c}$ some transient irregular bursts regime before the escape phenomena becomes robust. For a number of initial conditions, we have thus a distribution of values of critical noise around $\xi_{c}$ that is expected to become sharper as the number of initial conditions is increased. As a direct implication, the exponent obtained in our numerical simulations also varies within some range. For example, for the rotor map, if we choose $\xi_{c}=0.086+0.006$, we obtain $\alpha=1.5$, and for $\xi_{c}$ $=0.086-0.006$, we have $\alpha=2.0$. For the Hénon map, we ob-

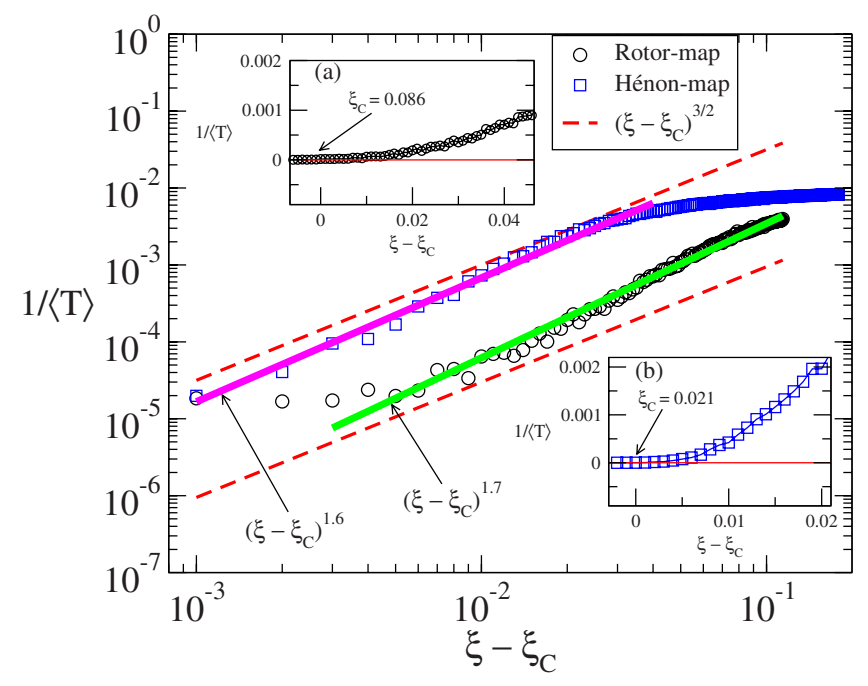

FIG. 3. (Color online) The inverse of the mean escape time scaling with amplitude of noise for map (12) (black circles) and for map (13) (blue squares). For each map, the values of mean escape time were obtained by iterating $10^{3}$ random orbits for each value of $\xi$. The dashed lines show the expected scaling $\left(\xi-\xi_{c}\right)^{3 / 2}$ and the thick continuous lines show the best fitting for the rotor map, $\alpha$ $\approx 1.7$, and for the Hénon map, $\alpha \approx 1.6$. In the insets, we show the transition. For $\xi<\xi_{c}$, we have $\langle T\rangle=\infty$, and the random orbits do not escape; therefore, $1 /\langle T\rangle=0$. For $\xi \geq \xi_{c}$, the escape time scales as Eq. (1). For the used parameters $\xi_{c}=0.086 \pm 0.006$ for the rotor map [inset (a)] and $\xi_{c}=0.021 \pm 0.002$ for the Hénon map [inset (b)].

tained $\alpha=1.3$ for $\xi_{c}=0.023$ and $\alpha=2.1$ for $\xi_{c}=0.019$.

Note also that, in principle, a much larger number of random orbits would be necessary for one to be able to observe a "perfect" power law. This is because most of the theoretical arguments used here, such as the convergence of time averages, are obtained in the asymptotic limit. Furthermore, we notice that for "large" values of $\left(\xi-\xi_{c}\right)$, meaning large holes, our simulated results differ appreciably from our theoretical prediction. This is due to the fact that $\mu_{c}\left(I_{\partial}\right)=\mu\left(I_{\partial}\right)$ is valid only for small leaks [20]. In addition, for large amplitude of noise, the dynamics is totally dominated by the noise and is not well described as a small perturbation around the deterministic motion. We note here that the exponential distribution reported in [4] for a particular case of bounded noise was obtained for values of $\xi$ much greater than the critical amplitude $\xi_{c}$, which is outside the range of validity of our theory.

As the last consideration, we want to call attention to the case where noise is applied on bifurcating systems [7,25,26]. In our approach we consider $\eta$ to be increasing. In Fig. 2, this corresponds to moving the parabola upward until it intersects the $\beta$ curve, when the transition to escape takes place. We can easily see that we should expect an equivalent transition to escape by moving $\beta$ instead, as a result of changing a bifurcating parameter while keeping the noise amplitude constant. Therefore, the exponent obtained for the case of bifurcating systems is expected to be the same as ours, and this is indeed the case $[7,25,26]$.

In conclusion, we have shown that the problem of escaping orbits from attractors due to the effect of bounded ran- 
dom noise can be thought as a closed system with a hole. We identify the subset of the phase space that is responsible for the escape and acts as a hole, and we show that the measure of this set determines the escape rate. We have shown that there is a critical amplitude of noise in order to such escape happens. When the amplitude is just above this critical value, we derived a universal power-law relation of escape time with respect to the amplitude of noise, in contrast with the case of Gaussian noise.

The authors are grateful to the anonymous referee for valuable suggestions to increase the value of this paper. A.P.S.d.M. and C.G. have been supported by the BBSRC under Grants No. BB-F00513X and No. BB-G010722.
[1] P. Hanggi, J. Stat. Phys. 42, 105 (1986).

[2] H. A. Kramers, Physica (Utrecht) 7, 284 (1940).

[3] P. Grasberger, J. Phys. A 22, 3283 (1989).

[4] S. Kraut, U. Feudel, and C. Grebogi, Phys. Rev. E 59, 5253 (1999).

[5] S. Kraut and U. Feudel, Phys. Rev. E 66, 015207 (2002).

[6] S. Kraut and C. Grebogi, Phys. Rev. Lett. 92, 234101 (2004).

[7] J. Demaeyer and P. Gaspard, Phys. Rev. E 80, 031147 (2009).

[8] P. D. Beale, Phys. Rev. A 40, 3998 (1989).

[9] N. Nagao, H. Nishimura, and N. Matsui, Neural Process. Lett. 12, 267 (2000); S. J. Schiff et al., Nature (London) 370, 615 (1994).

[10] O. Peters and K. Christensen, Phys. Rev. E 66, 036120 (2002); P. Bak, K. Christensen, L. Danon, and T. Scanlon, Phys. Rev. Lett. 88, 178501 (2002); M. Anghel, Chaos, Solitons Fractals 19, 399 (2004).

[11] L. Billings, E. M. Bollt, and I. B. Schwartz, Phys. Rev. Lett. 88, 234101 (2002); L. Billings and I. B. Schwartz, Chaos 18, 023122 (2008).

[12] V. Araujo, Ann. Inst. Henri Poincare, Anal. Non Lineaire 17, 307 (2000).

[13] C. Bonatti, L. J. Díaz, and M. Viana, Dynamics beyond Uniform Hyperbolicity (Springer, Berlin, 2005).

[14] The iteration of random maps can also be seen as an associated discrete time Markovian process having a family $\left\{p_{\xi}(\cdot \mid x): x\right.$ $\in U, \xi>0\}$ of transition probabilities, where every $p_{\xi}(\cdot \mid x)$ is supported on $U$, a $\xi$ neighborhood of $f(x)$.
[15] J.-P. Eckmann and D. Ruelle, Rev. Mod. Phys. 57, 617 (1985).

[16] More generally, for every continuous function $\varphi: M \rightarrow \mathbb{R}$.

[17] R. Mañé, Ergodic Theory of Differentiable Dynamics (Springer-Verlag, Berlin, 1987).

[18] G. Pianigiani and J. A. Yorke, Trans. Am. Math. Soc. 252, 351 (1979); M. F. Demers and L.-S. Young, Nonlinearity 19, 377 (2006); A. E. Motter and P. S. Letelier, Phys. Lett. A 285, 127 (2001); M. A. Sanjuán, T. Horita, and K. Aihara, Chaos 13, 17 (2003); L. A. Bunimovich and C. P. Dettmann, Phys. Rev. Lett. 94, 100201 (2005); Europhys. Lett. 80, 40001 (2007).

[19] H. Zmarrou and A. J. Homburg, Ergod. Theory Dyn. Syst. 27, 1651 (2007); Discrete Contin. Dyn. Syst., Ser. B 10, 719 (2008).

[20] E. G. Altmann and T. Tél, Phys. Rev. Lett. 100, 174101 (2008); Phys. Rev. E 79, 016204 (2009).

[21] M. Kac in Probability and Related Topics in Physical Sciences (Interscience Publishers, New York 1959), Chap. IV.

[22] Z. Liu, Y.-C. Lai, L. Billings, and I. B. Schwartz, Phys. Rev. Lett. 88, 124101 (2002).

[23] G. M. Zaslavskii, Phys. Lett. A 69, 145 (1978); B. Chirikov, Phys. Rep. 52, 263 (1979).

[24] C. S. Rodrigues, A. P. S. de Moura, and C. Grebogi, Phys. Rev. E 80, 026205 (2009).

[25] E. Ott, Chaos in Dynamical Systems, 2nd ed. (Cambridge University Press, Cambridge, England, 2002).

[26] J. C. Sommerer, E. Ott, and C. Grebogi, Phys. Rev. A 43, 1754 (1991). 